Check for updates

Cite this: Phys. Chem. Chem. Phys. 2022, 24, 568

Received 1st September 2021, Accepted 29th November 2021 DOI: 10.1039/d1cp04014f

rsc.li/pccp

\section{The excited-state dynamics of the radical anions of cyanoanthracenes $\dagger$}

\author{
Joseph S. Beckwith, (D) $\ddagger$ Alexander Aster§ and Eric Vauthey (D)*
}

The radical anion of 9,10-dicyanoanthracene (DCA) has been suggested to be a promising chromophore for photoredox chemistry, due to its nanosecond excited-state lifetime determined from indirect measurements. Here, we investigate the excited-state dynamics of the radical anion of three cyanoanthracenes, including $\mathbf{D C A}^{\bullet-}$, produced by photoinduced electron transfer in liquid using both pump-probe and pump-pump probe transient electronic absorption spectroscopy. All three excited radical ions are characterised by a 3-5 ps lifetime, due to efficient non-radiative deactivation to the ground state. The decay pathway most probably involves $D_{1} / D_{0}$ conical intersection(s), whose presence is favoured by the enhanced flexibility of the radical anions relative to their neutral counterparts. The origin of the discrepancy with the nanosecond lifetime of $\mathbf{D C A}^{-{ }^{-*}}$ reported previously is discussed. These very short lifetimes limit, but do not preclude, photochemical applications of the cyanoanthracene anions.

\section{Introduction}

Over the past few decades, open-shell ions in an electronic excited state have been drawing increasing attention in various areas of molecular sciences. Synthetic chemists have realised the enhanced chemical reactivity gained upon photoexcitation of radical ions, allowing for the reduction or oxidation of almost electrochemically inert compounds. ${ }^{1-7}$ In astrochemistry, fluorescence from radical cations of aromatic hydrocarbons has been suggested to be at the origin of emissive diffuse interstellar bands. ${ }^{8-11}$ In photoredox chemistry, the observation that bimolecular electron transfer (ET) between two neutral reactants is diffusion controlled even at driving forces at which Marcus inverted region is predicted, was explained by the formation of the ion-pair product in an electronic excited state. $^{12-15}$

Despite the relevance of photoexcited open-shell ions, not much is known about their dynamics. A large fraction of the radical cations investigated so far in liquids were found to have a very short excited-state lifetime, $<50$ ps. ${ }^{4,16-26}$ This was explained by the presence of easily accessible conical intersections between $\mathrm{D}_{1}$, the lowest electronic excited state, and $\mathrm{D}_{0}$, the ground state. $^{25,27-30}$ By contrast, nanosecond excited-state lifetimes were

Department of Physical Chemistry, University of Geneva, 30 Quai Ernest-Ansermet, CH-1211 Geneva 4, Switzerland. E-mail: eric.vauthey@unige.ch

$\dagger$ Electronic supplementary information (ESI) available: Experimental details, quantum-chemical calculations, and additional TA spectra. See DOI: 10.1039/ d1cp04014f.

\$ Present address: Department of Chemistry, Princeton University, Princeton, New Jersey 08544, USA.

$\S$ Present address: Solvias AG, Römerpark 2, 4303 Kaiseraugst, Switzerland. reported for several radical anions in liquids. ${ }^{31-34}$ However, these lifetimes were either deduced from indirect measurements or obtained from time-resolved fluorescence experiments, which are more sensitive to the presence of impurities and follow-up reaction products. To the best of our knowledge, the longest unambiguous excited-state lifetime determined so far amounts to $530 \mathrm{ps}$ and was measured with the radical anion of a perylene monoimide derivative produced electrochemically. ${ }^{35}$ However, most radical anions investigated until now in liquids have a significantly shorter excited-state lifetime. ${ }^{35-39}$

We report here on our investigation of the excited-state dynamics of the radical anion of three cyanoanthracenes (Fig. 1) in liquids using electronic transient absorption (TA) spectroscopy. These compounds were selected for their<smiles>N#Cc1c2ccccc2c(C#N)c2ccccc12</smiles>

9,10-Dicyanoanthracene DCA<smiles>N#Cc1ccc2c(C#N)c3ccccc3c(C#N)c2c1</smiles>

2,9,10-Tricyanoanthracene TrCA<smiles>N#Cc1ccc2c(C#N)c3cc([N+]#N)ccc3c(C#N)c2c1</smiles>

2,6,9,10-Tetracyanoanthracene

TCA

Fig. 1 Structures of the cyanoanthracenes. 
widespread use as electron-accepting chromophores in bimolecular ET studies. ${ }^{40-47}$ Combined with a strong electron donor, such as tetramethylphenylenediamine (TMPD), high ET driving forces, at which Marcus inverted region is expected, ${ }^{48}$ can be achieved. As the ET fluorescence quenching of these cyanoanthracenes by TMPD was found to be diffusion limited, ${ }^{45}$ it could possibly involve the electronic excited states of the ensuing radical ions. The radical cation of TMPD has been shown to have an excitedstate lifetime between 200 and $300 \mathrm{fs}^{25}$ Consequently, the cyanoanthracene radical anions might be better candidates for the detection of excited ionic product in a bimolecular ET process.

The radical anion of DCA produced electrochemically was initially reported to fluoresce in the 500-600 $\mathrm{nm}$ region with a $13.5 \mathrm{~ns}$ lifetime. ${ }^{32,49}$ This emission was later on shown to be due to a follow-up reaction product. ${ }^{50}$ A 4 ns excited-state lifetime was then deduced from bimolecular quenching measurements of $\mathbf{D C A}^{-{ }^{-*}}$ generated upon pulse radiolysis and subsequent photoexcitation with a $5 \mathrm{~ns}$ laser pulse. ${ }^{33}$ However, observation from our group that the photoexcitation of $\mathbf{D C A}^{\bullet}-$ produced upon bimolecular ET does not affect the free ion yield, suggests a significantly shorter excited-state lifetime. ${ }^{17}$

Here, stable solutions of $\mathbf{D C A}^{\bullet-}, \mathbf{T r C A}^{\bullet-}$ and $\mathbf{T C A}^{\bullet-}$ in acetonitrile were generated via a photoinduced ET reaction coupled with a scavenger counterion. Additionally, $\mathbf{D C A}^{\bullet-}$ and $\mathbf{T r C A}^{\bullet-}$ were also produced transiently upon photoinduced ET without a scavenger, and their excited-state dynamics were investigated using pump-pump-probe (PPP) TA.

The results presented here reveal unambiguously that the excited-state dynamics of all three radical anions is ultrafast, limiting their use in two-photon redox chemistry and complicating their detection in highly exergonic ET reactions.

\section{Experimental}

\subsection{Samples}

9,10-Dicyanoanthracene (DCA) was purchased from Kodak and recrystallised before use. 2,9,10-Tricyanoanthracene (TrCA) and 2,6,9,10-tetracyanoanthracene (TCA) were synthesised as described previously. ${ }^{51}$ All measurements were performed in acetonitrile (ACN) (Roth, Rotidry, $\geq 99.9 \%$ ), except one that was carried out in $N, N$-dimethylformamide (DMF, Sigma-Aldrich, puriss.).

The radical anions were generated upon photoinduced ET between the cyanoanthracenes and triethylamine (TEA, Fluka) in the presence of tetra- $n$-butylammonium dihydrogen phosphate (TBADHP, Alfa Aesar) in ACN (Section S1, ESI $\dagger$ ). ${ }^{52}$ TEA was distilled before use and kept under argon. The principle of this procedure, whilst not fully established, ${ }^{52}$ appears to rest on the stabilisation of the radical anion via association with a large organic salt (i.e. TBADHP). The radical anions of DCA and TrCA were also generated upon ET quenching with either 1,2,6-trimethylbenzene (TMB, Fluka, puriss.) or mesitylene (MES, Fluka, puriss.) in ACN or DMF.

\subsection{Transient absorption spectroscopy}

Transient absorption (TA) measurements were performed with a setup described in ref. 53 and based on an amplified
Ti:Sapphire system (Solstice Ace, Spectra-Physics), producing 35 fs pulses centred at $800 \mathrm{~nm}$ with a $5 \mathrm{kHz}$ repetition rate (Section S2, ESI $\dagger$ ). In brief, the pump pulses were produced by a TOPAS-Prime combined with a NirUVis module (Light Conversion), and were compressed to 60-100 fs at the sample position. The pump intensity on the sample was $\sim 0.15-0.75 \mathrm{~mJ} \mathrm{~cm} \mathrm{~cm}^{-2}$. Probing was achieved from about 320 to $750 \mathrm{~nm}$ using white light pulses generated in a $3 \mathrm{~mm} \mathrm{CaF}_{2}$ plate. The polarisation of the pump pulses was at magic angle with respect to that of the probe pulses. The sample cell was $1 \mathrm{~mm}$ thick and the instrument response function had a full width at half maximum varying between 80 and $350 \mathrm{fs}$, depending on the probe wavelength.

The principle of the pump-pump-probe (PPP) experiment used for investigating the excited-state dynamics of $\mathbf{D C A}^{\bullet-}$ is described in ref. 54. The setup was the same as that used for the TA measurement except for the use of a first pump pulse at $400 \mathrm{~nm}$, produced by frequency doubling part of the output pulses of the Ti:Sapphire amplifier. This actinic pulse was used to excite DCA and trigger the ET between DCA* and an electron donor. Further details can be found in ref. 54 .

\subsection{Quantum-chemical calculations}

All calculations were carried out at the density functional theory (DFT) or time-dependent (TD) DFT level in the gas phase, using both the long-range corrected hybrid CAM-B3LYP functional ${ }^{55}$ with the augmented cc-pVDZ basis set and the B3LYP functional $^{56}$ with the $6-31+G^{*}$ basis set, as implemented in the Gaussian16 (Rev. B) software. ${ }^{57}$ Both approaches gave qualitatively similar results.

\section{Results}

\subsection{Stationary spectroscopy}

The absorption spectra of DCA, TrCA and TCA in ACN are shown in Fig. 2.

Upon addition of TEA + TBADHP and irradiation at $405 \mathrm{~nm}$, the absorption bands of the anions appear in the electronic spectrum. Fig. 3 shows the absorption spectra of all three anions obtained after about $1 \mathrm{~h}$ irradiation and after subtraction of the residual absorption band of the neutrals. The absorption

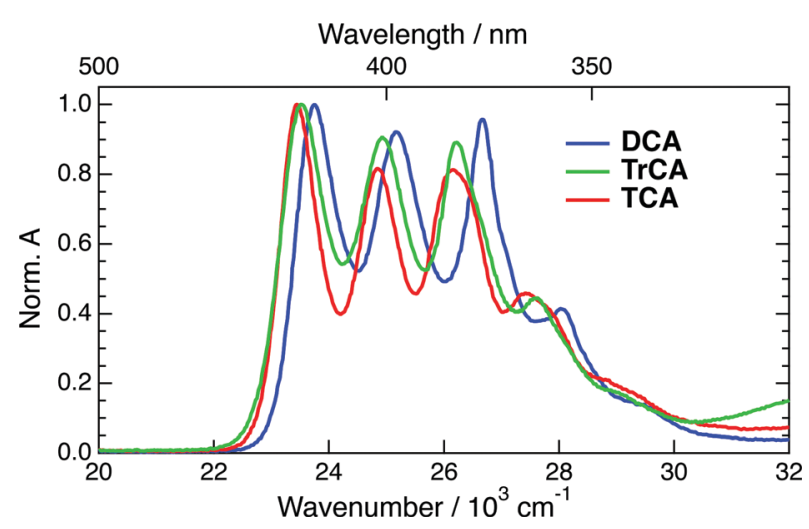

Fig. 2 Stationary absorption spectra of DCA, TrCA and TCA in acetonitrile. 


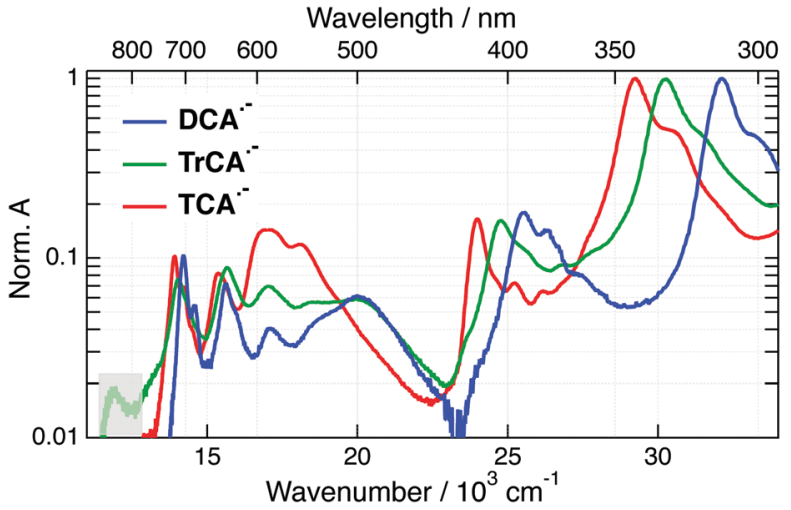

Fig. 3 Stationary absorption spectra of $\mathbf{D C A}^{\bullet}-$, $\operatorname{TrCA}^{\boldsymbol{\bullet}^{-}}$and $\mathrm{TCA}^{\boldsymbol{*}^{-}}$in the presence of TEA + TBADHP in acetonitrile after $405 \mathrm{~nm}$ irradiation and subtraction of the absorption bands of the neutral precursor. The signal in the shaded area is attributed to an artefact.

spectra of $\mathbf{D C A}^{\bullet-}$ and $\mathbf{T C A}^{\bullet-}$ coincide with those published previously. $^{33,58,59}$ The similarity of the spectrum obtained with TrCA to the $\mathbf{D C A}^{\bullet-}$ and $\mathbf{T C A}^{\bullet-}$ spectra gives good confidence that it originates from $\operatorname{TrCA}^{\bullet-}$. The minor feature at $12000 \mathrm{~cm}^{-1}$ is attributed to an artefact as its amplitude relative to the other bands is not constant during irradiation (Section S3, ESI $\dagger$ ).

The absorption spectra of the three radical ions present a similar shape, with all bands shifting to lower energy upon increasing the number of cyano groups. For DCA $^{\bullet-}$, a molar absorption coefficient of $8.1 \times 10^{3} \mathrm{M}^{-1} \mathrm{~cm}^{-1}$ at $14100 \mathrm{~cm}^{-1}$ (705 $\mathrm{nm}$ ) was reported. ${ }^{58,60}$ Given the similarity in the shapes the radical anion spectra, the molar absorption coefficients at the peaks around $14000 \mathrm{~cm}^{-1}$ should not differ very significantly for $\mathbf{T r C A}^{\bullet-}$ and $\mathbf{T C A}^{\bullet-}$. This coefficient corresponds to a transition dipole moment of $\sim 1.7 \mathrm{D}$ and an oscillator strength of 0.02 (Section S3, ESI $\dagger$ ).

The lowest energy absorption predicted from TD-DFT calculations is around $2 \mathrm{eV}(620 \mathrm{~nm})$ for all three anions with an oscillator strength between 0.02 and 0.06 (Section S4 $\dagger$ ). This absorption corresponds to the $\mathrm{D}_{1} \leftarrow \mathrm{D}_{0}$ transition for $\mathbf{D C A}^{\bullet-}$ and $\operatorname{TrCA}^{\bullet-}$, and to the $\mathrm{D}_{2} \leftarrow \mathrm{D}_{0}$ transition for $\mathbf{T C A}^{\bullet-}$. For the latter, the calculated $\mathrm{D}_{1} \leftarrow \mathrm{D}_{0}$ transition has no oscillator strength and its energy is less than $0.1 \mathrm{eV}$ below $\mathrm{D}_{2} \leftarrow \mathrm{D}_{0}$. In the case of $\mathbf{D C A}^{\bullet-}$, a forbidden $\mathrm{D}_{2} \leftarrow \mathrm{D}_{0}$ transition less than $0.2 \mathrm{eV}$ above $\mathrm{D}_{1} \leftarrow \mathrm{D}_{0}$ is predicted. For $\operatorname{TrCA}^{\bullet-}$, this second transition is also close to $D_{1} \leftarrow D_{0}$ but is allowed. This difference can be explained by the lower symmetry of $\operatorname{TrCA}^{\bullet-}$, i.e. the lack of a centre of inversion, compared to $\mathbf{D C A}^{\bullet-}$ and TCA $^{-}$, in agreement with the Laporte rules. ${ }^{61,62}$ As illustrated in Fig. S3 (ESI $\dagger$ ), these calculated transitions are in reasonably good agreement with the experiment.

\subsection{Transient absorption spectroscopy}

3.2.1 Neutral cyanoanthracenes. The TA measurements with all three radical anions were performed upon $710 \mathrm{~nm}$ excitation, corresponding to the lowest-energy absorption band (Fig. 3). As shown in Fig. 2, the neutral cyanoanthracenes do not absorb at this wavelength. Therefore, although both the anionic and the neutral forms coexist after irradiation in the presence of TEA and TBADHP, only the radical anions are excited. Nevertheless, the presence of the $S_{1}$ state of the neutral upon photoexcitation of the radical anion has been reported with the radical anion of perylene produced upon photoinduced ET with dimethylaniline. ${ }^{54}$ In this case, the charge recombination between the excited perylene anion and the aniline radical cation lead to the repopulation of neutral perylene in the $S_{1}$ state. Therefore, in order to detect the occurrence of a similar process upon photoexcitation of the cyanoanthracene anions, it is necessary to be able to identify the spectral features of their neutral precursors in the $S_{1}$ state.

TA spectra measured $10 \mathrm{ps}$ after $400 \mathrm{~nm}$ excitation of all three neutrals in ACN are shown in Fig. 4. The transient spectra of the cyanoanthracenes are relatively similar and consist mainly of three excited-state absorption bands. The stimulated $S_{1} \rightarrow S_{0}$ emission appears as a weak negative band around $475 \mathrm{~nm}$, and the bleach of the $\mathrm{S}_{1} \leftarrow \mathrm{S}_{0}$ absorption is only visible around $380 \mathrm{~nm}$ with DCA. The relative weakness of the bleach points to a large absorption coefficient of the overlapping ESA band. These TA data were analysed globally assuming a series of successive exponential steps with increasing time constants. ${ }^{63,64}$ The resulting evolution-associated difference absorption spectra (EADS) and the corresponding time constants are shown in Fig. S6 (ESI $\dagger$ ). Apart from minor spectral shifts during the first $20 \mathrm{ps}$, which can be assigned to vibrational/solvent relaxation, the dynamics are dominated by the nanosecond decay of the excited-state population.

3.2.2 DCA $^{\bullet-}$. TA spectra measured with DCA $^{\bullet-}$ in the presence of TEA/TBADHP in ACN are illustrated in Fig. 5A. Directly after $710 \mathrm{~nm}$ excitation, the spectra consist of two positive bands, one centred at $460 \mathrm{~nm}$ and the other at $370 \mathrm{~nm}$, and of ground-state bleach features above $550 \mathrm{~nm}$ and at $400 \mathrm{~nm}$. The bleach associated with the $400 \mathrm{~nm}$ band is hardly visible due to the presence of an overlapping positive band. The $460 \mathrm{~nm}$ band decays entirely within about $5 \mathrm{ps}$, whereas the $370 \mathrm{~nm}$ band increases concurrently and the bleach decreases partially. Afterwards, all spectral features decay entirely in a few tens of ps. These spectral dynamics could be adequately

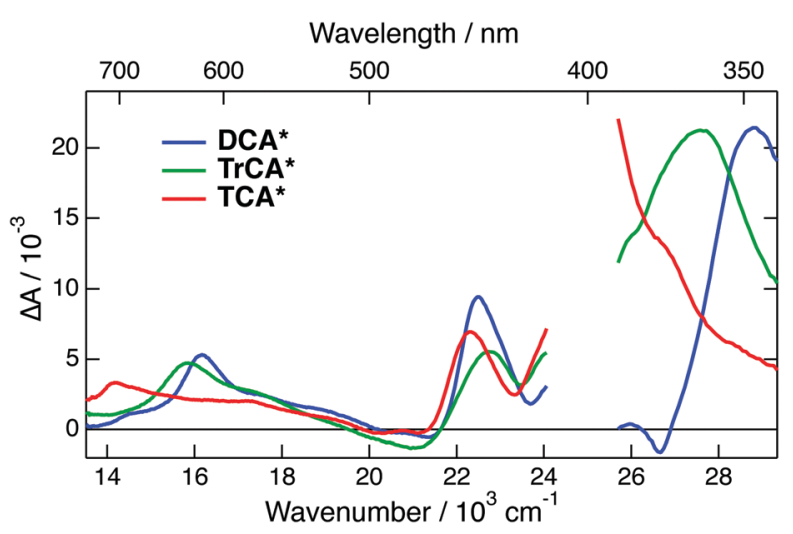

Fig. 4 Transient absorption spectra measured 10 ps after 400 nm excitation of the cyanoanthracenes in acetonitrile. 


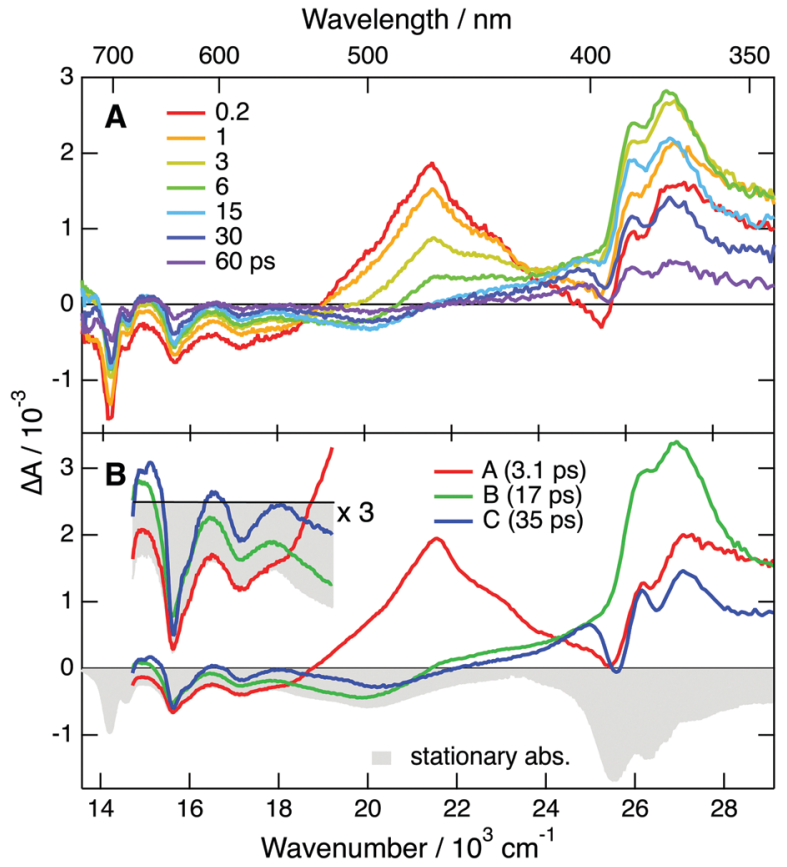

Fig. 5 (A) Transient absorption spectra measured various time delays after $710 \mathrm{~nm}$ excitation of $\mathbf{D C A}^{\bullet-}$ generated upon continuous irradiation of a DCA/TEA/TBADHP solution. (B) Evolution-associated difference absorption spectra and time constants obtained from a global analysis of the transient absorption data assuming three successive exponential steps, $\mathrm{A} \rightarrow \mathrm{B} \rightarrow \mathrm{C} \rightarrow \mathrm{D}, \mathrm{D}$ being the ground state, and negative stationary absorption spectrum (bottom).

reproduced by global analysis assuming three consecutive steps $\left(\mathrm{A} \rightarrow \ldots \rightarrow \mathrm{D}\right.$, where $\mathrm{D}$ is the $\mathrm{D}_{0}$ state) with the EADS and time constants shown in Fig. 5B. Given the timescale on which the spectral changes take place, non-equilibrium, hence nonexponential dynamics can be expected. ${ }^{65,66}$ Consequently, the EADS cannot be attributed to a well-defined state/species but may contain contributions from different transients. Similarly, the corresponding time constants should only be considered as representative time-scales. The first spectrum, EADS A, can be largely attributed to the photopopulated excited state, which, according to the quantum-chemical calculations, should be the $\mathrm{D}_{1}$ state. The last spectrum, EADS C presents positive spectral features on the low-energy side of the ground-state bleach at $400 \mathrm{~nm}$ and above $550 \mathrm{~nm}$, which are characteristic of a vibrationally hot ground state. ${ }^{67-69}$ The interpretation of EADS $\mathrm{B}$ is more ambiguous. If the photo-populated state (EADS A) is the $\mathrm{D}_{1}$ state, then EADS $\mathrm{B}$ should mostly reflect spectral features of the vibrationally hot $D_{0}$ state. More than $1.7 \mathrm{eV}$ is released non-radiatively upon $\mathrm{D}_{1} \rightarrow \mathrm{D}_{0}$ internal conversion. Dissipation of such a large amount of energy via vibrational relaxation occurs on multiple timescales, ${ }^{67,70-72}$ and thus, EADS $\mathrm{B}$ and $\mathrm{C}$ could correspond to the ground state at different stages of its thermal equilibration. The $\mathrm{B} \rightarrow \mathrm{C}$ and $\mathrm{C} \rightarrow \mathrm{D}$ time constants are consistent with values reported previously. ${ }^{67,73-76}$ Interpretation of the $\mathrm{B} \rightarrow \mathrm{C}$ step as vibrational relaxation is supported by the presence of hot ground-state features above $550 \mathrm{~nm}$ already in EADS B (Fig. 5B, inset). Attribution of the
$375 \mathrm{~nm}$ band to a hot ground-state feature is not so straightforward. However, as shown in Fig. 3, $\mathbf{D C A}^{\bullet-}$ exhibits a very intense absorption band peaking at $312 \mathrm{~nm}$. Consequently, the hot ground-state features associated to this band can be expected to be at least five times as intense that those related to the $400 \mathrm{~nm}$ band (Fig. S7, ESI $\dagger$ ). Consequently, the amplitude of this hot-ground state band could be large enough to hide the bleach of the $400 \mathrm{~nm}$ band. The presence of the $375 \mathrm{~nm}$ band already in EADS A could reflect the nonexponential character of the $\mathrm{D}_{1} \rightarrow \mathrm{D}_{0}$ internal conversion. Among the alternative interpretation of the $375 \mathrm{~nm}$ band, DCA in both ground and excited state can be ruled out as this band is absent from their absorption spectra (Fig. 2 and 4).

In principle, this band could also arise from a follow-up species produced upon reaction between DCA $^{\bullet-*}$ with TEA or TBADHP. As shown in Fig. S8 (ESI $\dagger$ ), the excited-state dynamics of $\mathbf{D C A}^{--}$is independent of the concentration of TEA, ruling out the quenching of DCA $^{\bullet^{-*}}$ by TEA.

To further explore occurrence of follow-up reactions, $\mathbf{D C A}^{\bullet-}$ was produced by photoinduced ET with a different electron donor, 1,2,6-trimethylbenzene (TMB), and without TBADHP. Due to the absence of the scavenger TBADHP, the ion population decays on the microsecond timescale by homogeneous recombination. ${ }^{77}$ Therefore, the excited-state dynamics of DCA $^{--}$was measured by PPP TA. The TA spectra obtained at different time delays after $400 \mathrm{~nm}$ excitation of DCA with $1 \mathrm{M}$ TMB in ACN (Fig. S9, ESI $\dagger$ ) show distinctly the transformation of DCA $^{*}$ into $\mathbf{D C A}^{\mathbf{}}-$. For the PPP measurements, a second pump pulse at $710 \mathrm{~nm}$ was applied $1 \mathrm{~ns}$ after the first pump pulse at $400 \mathrm{~nm}$, to excite $\mathbf{D C A}^{\bullet-}$, and probing was performed at different time delays, $\Delta t_{23}$, after the second pump pulse. The resulting PPP spectra correspond to the difference absorption spectra with and without the second pump pulse. Consequently, they can be readily compared with the conventional pump-probe spectra of $\mathbf{D C A}^{\bullet-}$ depicted in Fig. 5. Fig. 6 illustrates resulting PPP spectra as well as the EADS and corresponding time constants obtained from a global analysis of the PPP data. Because of the small transient absorbance at $710 \mathrm{~nm}$ induced by the first pump pulse, the PPP signal is weak and, consequently, two successive exponential steps $\left(\mathrm{A}^{\prime} \rightarrow \mathrm{B}^{\prime} \rightarrow \mathrm{C}^{\prime}, \mathrm{C}^{\prime}\right.$ being $\left.\mathrm{D}_{0}\right)$ are sufficient to reproduce the data.

The first EADS, $\mathrm{A}^{\prime}$, is very similar to EADS A (Fig. 5B) with the presence of the $460 \mathrm{~nm}$ band, and decays with a similar time constant of $2.8 \mathrm{ps}$. On the other hand, EADS $\mathrm{B}^{\prime}$ contains features present in both EADS B and $\mathrm{C}$ and decays in 36 ps. The signal-to-noise ratio of these PPP TA data is not sufficient to resolve the intermediate $\mathrm{B} \rightarrow \mathrm{C}$ step with a $19 \mathrm{ps}$ time constant. The similarity of the EADS and time constants obtained from the pump-probe and PPP TA measurements reveals that the excitedstate dynamics of $\mathbf{D C A}^{\bullet-}$ depends neither on the nature of the electron donor and nor on the presence of the TBADHP scavenger.

According to ref. 5, the oxidation potential of the DCA/ DCA $^{\bullet-*}$ couple amounts to $-3.2 \mathrm{~V} v s$. SCE. Given the reduction potential of liquid acetonitrile of $-2.8 \mathrm{~V} v s$. SCE, ${ }^{78}$ an ET from to the solvent should be considered. To explore this possibility, PPP measurements were repeated in DMF, whose reduction 


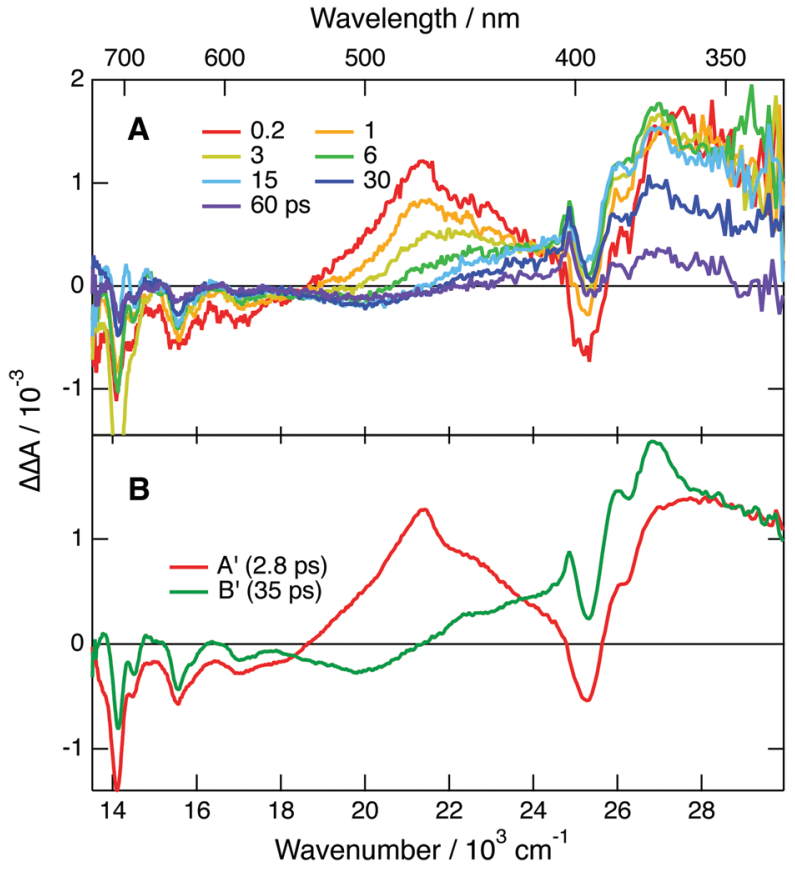

Fig. 6 (A) PPP spectra measured with DCA and $1 \mathrm{MTMB}$ in ACN at different delays, $\Delta t_{23}$, after the second pump pulse at $710 \mathrm{~nm}$. The delay between the actinic pulse at $400 \mathrm{~nm}$ and the $710 \mathrm{~nm}$ pulse was $1 \mathrm{~ns}$. (B) Evolution-associated difference absorption spectra and time constants obtained from a global analysis of the PPP data assuming two successive exponential steps $A^{\prime} \rightarrow B^{\prime} \rightarrow C^{\prime}, C^{\prime}$ being the $D_{0}$ state.

potential amounts to $-3.0 \mathrm{~V}$ vs. SCE. ${ }^{78}$ As shown in Fig. S13 (ESI $\dagger$ ), the lifetime of the $460 \mathrm{~nm}$ band is exactly the same in both ACN and DMF, ruling out ET quenching of by the solvent. In fact, the $-3.2 \mathrm{~V}$ oxidation potential of reported in ref. 5 was calculated assuming an excited-state energy of $2.4 \mathrm{eV}$, itself taken from the absorption and emission spectra presented in ref. 49, that were later on shown to be associated with a follow-up reaction product of $\mathbf{D C A}^{\bullet-}$. With a $\mathrm{D}_{1}$ state energy of $1.75 \mathrm{eV}$, deduced from the absorption spectrum shown in Fig. 3, the oxidation potential of the $\mathbf{D C A} / \mathbf{D C A}^{\bullet-*}$ couple is only about $-2.6 \mathrm{~V}$ vs. SCE. This is insufficient for $\mathbf{D C A}^{\mathbf{0}^{-*} \text { to }}$ undergo ET with ACN and DMF, in agreement with the PPP results. Therefore, the $375 \mathrm{~nm}$ TA band cannot be assigned to a follow-up reaction product of $\mathbf{D C A}^{\bullet-*}$.

The quantum-chemical calculations in the gas phase predict that the $\mathrm{D}_{2}$ state of $\mathbf{D C A} \mathbf{A}^{--}$at the ground-state geometry is less than $0.2 \mathrm{eV}$ above the $\mathrm{D}_{1}$ state (Fig. S5, ESI $\dagger$ ). Given this small difference of energy, one cannot exclude that these two states are actually inverted, and that optical excitation populates the $\mathrm{D}_{2}$ state, as the $\mathrm{D}_{1} \leftarrow \mathrm{D}_{0}$ transition is forbidden. In this case, the EADS A and $\mathrm{B}$ could be attributed to the $\mathrm{D}_{2}$ and $\mathrm{D}_{1}$ state, respectively. According to this scenario, the $2.8 \mathrm{ps} \mathrm{A} \rightarrow$ B step would correspond to the $\mathrm{D}_{2} \rightarrow \mathrm{D}_{1}$ internal conversion and the $\mathrm{D}_{1}$ state lifetime would amount to $19 \mathrm{ps}$. Although this hypothesis cannot be totally ruled out, it is quite improbable since it would imply a surprisingly slow $\mathrm{D}_{2} \rightarrow \mathrm{D}_{1}$ internal conversion. Based on this, the above hypothesis with the lifetime of the $\mathrm{D}_{1}$ state amounting to about 3 ps seems the most plausible. This assignment of EADS $\mathrm{B}$ and $\mathrm{C}$ to the hot $\mathrm{D}_{0}$ state is further supported by the spectra measured with $\operatorname{TrCA}^{\bullet-}$ and $\mathbf{T C A}^{\bullet-}$ discussed below with which these hot ground state features are clearer.

3.2.3 $\operatorname{TrCA}^{\bullet-}$. Although the quantum-chemical calculations do not predict any low-energy forbidden transition, interpretation of the results obtained with $\mathbf{T r C A}^{\bullet-}$ is also not totally obvious. TA spectra measured at different time delays after $710 \mathrm{~nm}$ excitation of $\mathbf{T r C A}^{\bullet-}$ prepared upon irradiation of a TrCA/TEA/TBADHP mixture in ACN are illustrated in Fig. 7, together with the results of a global analysis assuming four successive exponential steps $\left(\mathrm{A} \rightarrow \ldots \rightarrow \mathrm{E}\right.$, where $\mathrm{E}$ is the $\mathrm{D}_{0}$ state). The earliest spectra are dominated by an intense positive band peaking at $380 \mathrm{~nm}$. Additionally, ground-state bleach, which is visible above $475 \mathrm{~nm}$, is superimposed with positive features above $\sim 650 \mathrm{~nm}$. The onset of the ground-state bleach associated with the intense absorption band of $\mathbf{T r C A}^{\bullet-}$ at $330 \mathrm{~nm}$ is just visible at the shortest wavelength. During the first $10 \mathrm{ps}$, the $380 \mathrm{~nm}$ band decays and transforms into a band with a maximum below $340 \mathrm{~nm}$ and a red edge extending to $440 \mathrm{~nm}$. In parallel, the ground-state bleach between 500 and $600 \mathrm{~nm}$ decreases significantly. Finally, the residual transient spectrum shows a sawtooth-shaped band at $400 \mathrm{~nm}$ and decays completely within $60 \mathrm{ps}$.

All positive features in EADS C and D are located on the lowenergy side of bleaches. Based on this and on the associated

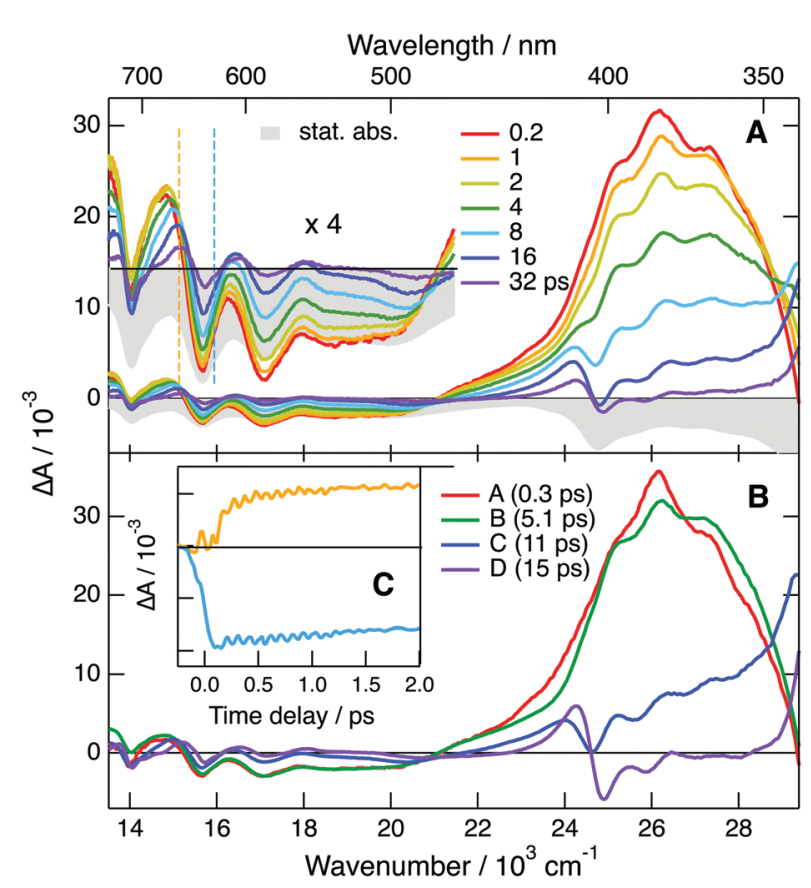

Fig. 7 (A) Transient absorption spectra measured various time delays after $710 \mathrm{~nm}$ excitation of $\operatorname{TrCA}^{\bullet-}$ generated upon continuous irradiation of a TrCA/TEA/TBADHP solution, and negative stationary absorption spectrum. (B) Evolution-associated difference absorption spectra and time constants obtained from a global analysis of the transient absorption data assuming four successive exponential steps, $A \rightarrow \ldots \rightarrow E$, E being the ground state. (C) Time profiles of the transient absorption at the wavenumbers shown by the dashed lines in (A). 
time constants, these spectra can be safely assigned to the hot ground state. The positive band at $380 \mathrm{~nm}$, dominating EADS A and $\mathrm{B}$, is also on the red side of the bleach at $330 \mathrm{~nm}$, and could thus also originate from the hot ground state, but at an earlier stage of thermal equilibration. However, the area of this band is larger than that expected for the $330 \mathrm{~nm}$ bleach (Fig. S11, ESI $\dagger$ ). If this band were really due to the hot ground state, this would imply that the oscillator strength of this transition increases very significantly with the vibrational temperature of the molecule. While not totally impossible, such an effect would be a violation of the Condon approximation. ${ }^{79}$ Therefore, the assignment of this band to a $\mathrm{D}_{n} \leftarrow \mathrm{D}_{1}$ absorption seems more plausible. In this case, the $\mathrm{A} \rightarrow \mathrm{B}$ and $\mathrm{B} \rightarrow \mathrm{C}$ steps mostly reflect the equilibration of the Franck-Condon $\mathrm{D}_{1}$ state and the decay of the $\mathrm{D}_{1}$ state by internal conversion, respectively. The excited-state lifetime of $\operatorname{TrCA}^{\bullet-}$ amounts, thus, to $\sim 5$ ps.

Additionally, the time dependence of the TA intensity above $550 \mathrm{~nm}$ exhibits a distinct oscillation during the first ps after excitation (Fig. 7C). As illustrated by the contour plot in Fig. S15 (ESI $\dagger$ ), a node can be observed at $\sim 640 \mathrm{~nm}$, the maximum of the second lowest-energy absorption band of $\operatorname{TrCA}^{\bullet-}$. One can thus conclude that this oscillation arises from a coherent vibrational wavepacket in the $\mathrm{D}_{0}$ state produced via a stimulated Raman process. ${ }^{80-82}$ Fourier transformation of the oscillation points to a vibrational mode at $317 \mathrm{~cm}^{-1}$ (Fig. S16, ESI $\dagger$ ). Quantum-chemical calculations of $\mathbf{T r C A}^{\bullet-}$ in the ground state predict a Raman active vibrational mode at $329 \mathrm{~cm}^{-1}$, which is associated with an in-plane distortion of the anthracene core (Fig. S17, ESI $\dagger$ ).

Very similar dynamics were observed upon PPP TA measurements, where $\mathbf{T r C A}^{\bullet-}$ was generated upon photoinduced ET between TrCA in the $S_{1}$ state and 1 M MES in ACN (Fig. S12 and $\mathrm{S} 14 \mathrm{~A}, \mathrm{ESI} \dagger)$. The PPP data were analysed assuming two successive exponential steps $\left(\mathrm{A}^{\prime} \rightarrow \mathrm{B}^{\prime} \rightarrow \mathrm{C}^{\prime}\right)$. The resulting EADS $\mathrm{A}^{\prime}$ resembles those of $\mathrm{A}$ and $\mathrm{B}$ shown in Fig. 7B, whereas EADS $\mathrm{B}^{\prime}$ shows the characteristic feature of the hot ground state (Fig. S14B, ESI $\dagger$ ).

3.2.4 $\mathbf{T C A}^{\bullet-}$. TA spectra measured at different time delays after $710 \mathrm{~nm}$ excitation of $\mathbf{T C A}^{\bullet}$ - prepared by irradiation of a TCA/TEA/TBADHP mixture in ACN are presented in Fig. 8, together with the EADS obtained assuming four successive exponential steps $\left(\mathrm{A} \rightarrow \ldots \rightarrow \mathrm{E}\right.$, where $\mathrm{E}$ is the $\mathrm{D}_{0}$ state). Although the time constants related to the $\mathrm{C} \rightarrow \mathrm{D} \rightarrow$ E steps are close to each other, the data could not be satisfactorily reproduced with only three steps. The earliest TA spectra show two positive bands, one peaking at $535 \mathrm{~nm}$ with a distinct vibronic structure, and another with a maximum at about $360 \mathrm{~nm}$ and a broad shoulder. This second band is located on the low-energy side of an intense ground-state bleach around $340 \mathrm{~nm}$. Weaker ground-state bleach features are also visible above $550 \mathrm{~nm}$. The $535 \mathrm{~nm}$ band decays entirely within $400 \mathrm{fs}$, whereas the shape of the other band changes considerably, with the maximum moving to $410 \mathrm{~nm}$. Thereafter, this band transforms on a 10-15 ps timescale to a band with the maximum continuously shifting towards shorter wavelength. This transformation is accompanied by a decrease of the

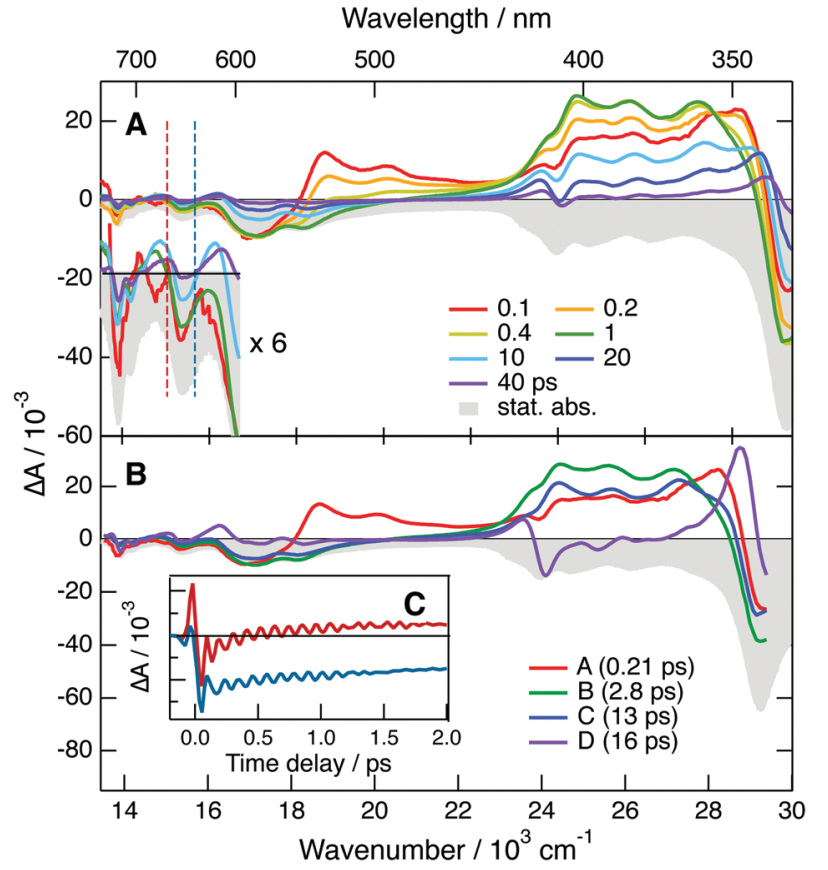

Fig. 8 (A) Transient absorption spectra measured various time delays after $710 \mathrm{~nm}$ excitation of $\mathrm{TCA}^{{ }^{-}-}$generated upon continuous irradiation of a TCA/TEA/TBADHP solution, and negative stationary absorption spectrum. (B) Evolution-associated difference absorption spectra and time constants obtained from a global analysis of the transient absorption data assuming four successive exponential steps, $A \rightarrow \ldots \rightarrow E$, E being the ground state. (C) Time profiles of the transient absorption at the wavenumbers shown by the dashed lines in (A)

$340 \mathrm{~nm}$ ground-state bleach as well as by the appearance of positive bands between the ground-state bleach features above $550 \mathrm{~nm}$. These bands, together with all the other spectral features, decay entirely within about $50 \mathrm{ps}$.

The quantum-chemical calculations performed with $\mathbf{T C A}^{\bullet}$ predict that optical excitation in the lowest-energy absorption band populates the $D_{2}$ state, because the $D_{1} \leftarrow D_{0}$ transition has no oscillator strength. Based on this, the $535 \mathrm{~nm}$ band observed at the earliest time delays can be attributed to the $\mathrm{D}_{2}$ state. Consequently, the $210 \mathrm{fs}$ time constant obtained from the global analysis can be assigned to the $D_{2} \rightarrow D_{1}$ internal conversion. The order of magnitude of this time constant agrees with that expected for the excited-state lifetime of an upper-excited state. Whereas EADS A is mostly due to the $\mathrm{D}_{2}$ state, EADS B, which is dominated by the positive band with a maximum at $410 \mathrm{~nm}$, should correspond to the $\mathrm{D}_{1}$ state. However, given that this band is located on the red side of the strong bleach at $340 \mathrm{~nm}$, it is not clear whether it can really be interpreted as a $\mathrm{D}_{n} \leftarrow \mathrm{D}_{1}$ transition or it is dominated by hot-ground state absorption. The first interpretation implies that the lifetime of the $\mathrm{D}_{1}$ state amounts to $2.8 \mathrm{ps}$. According to the second interpretation, the $\mathrm{D}_{1} \rightarrow \mathrm{D}_{0}$ internal conversion is significantly faster and this time constants reflects mostly the early stage of vibrational relaxation. However, the fact that the ground-state bleach features do not decrease significantly, during the $\mathrm{A} \rightarrow \mathrm{B}$ step is rather in favour of the first interpretation. 
Additionally, the relatively large area of the $410 \mathrm{~nm}$ band does not point to an entirely hot ground state feature. As stated above, nonequilibrium and, thus, non-exponential dynamics can be expected for processes occurring on this timescale. Consequently, additionally to the $\mathrm{D}_{1}$ state, EADS $\mathrm{B}$ could also contain a non-negligible contribution from the hot ground state. On the other hand, the last two steps, $\mathrm{C} \rightarrow \mathrm{D} \rightarrow \mathrm{E}$, can unambiguously be attributed to the equilibration of the hot ground state.

Similarly to $\operatorname{TrCA}^{--}$an oscillation can also be observed at early time in the TA data above $550 \mathrm{~nm}$ (Fig. 8C). Here, the node is at $650 \mathrm{~nm}$, the maximum of the second lowest-energy absorption band of TCA $^{\bullet-}$ (Fig. S18, ESI $\dagger$ ). This oscillation can thus also be attributed to a coherent vibrational wavepacket in the $\mathrm{D}_{0}$ state. This oscillation is at $307 \mathrm{~cm}^{-1}$ (Fig. S19, ESI $\dagger$ ), whereas quantum-chemical calculations of $\mathbf{T C A}^{--}$in the ground state predict a Raman active vibrational mode at $300 \mathrm{~cm}^{-1}$, which is also associated with in-plane distortion of the anthracene core (Fig. S20, ESI $\dagger$ ). The slightly lower frequency of this mode in $\mathbf{T C A}^{--}$relative to $\mathbf{T r C A}^{--}$can be explained by the presence of the $\mathrm{C} \equiv \mathrm{N}$ group in 6 position instead of a $\mathrm{H}$ atom. Because of the absence of $\mathrm{C} \equiv \mathrm{N}$ groups in the 2 and 6 positions, this normal mode is lacking in $\mathbf{D C A}^{\bullet-}$. For this anion, the calculations predict a Raman-active vibration at $358 \mathrm{~cm}^{-1}$. This corresponds to a vibrational period of $90 \mathrm{fs}$, which is probably just too short to be properly resolved with the instrument response function of the TA setup. The absence of vibrational coherence in the TA data measured with DCA $^{\bullet-}$ is, thus, not surprising.

\section{Discussion}

Fig. 9 summarises the most probable deactivation pathways following photoexcitation of the three radical anions, based on the above-described interpretation of the TA data. For $\mathbf{D C A}^{\bullet-}$ and $\operatorname{TrCA}^{--}$, the $\mathrm{D}_{1}$ state is directly populated upon $710 \mathrm{~nm}$ excitation. As the $D_{1} \leftarrow D_{0}$ transition is predicted to be forbidden for $\mathbf{T C A}^{\bullet-}$, the $\mathrm{D}_{1}$ state is populated upon internal conversion from the $\mathrm{D}_{2}$ state with a $\sim 200$ fs time constant. The $\mathrm{D}_{1}$ state of all three radical anions is characterised by a very short lifetime, between 3 and 5 ps, due to the existence of very efficient non-radiative decay channel(s). As this internal

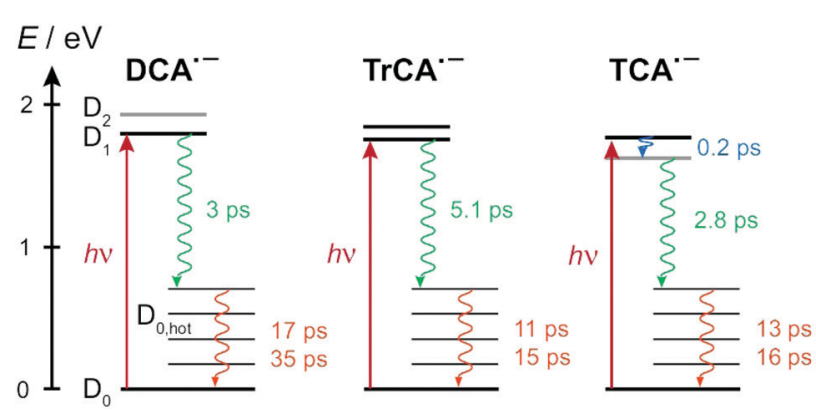

Fig. 9 Energy-level diagrams illustrating the most probable decay pathways of the photopopulated state of the radical anions, with the associated time constants. The grey lines designate electronic states that are dark with respect to the ground state. conversion is faster than vibrational relaxation, the decay of the ground-state bleach features occurs on a slower timescale through the equilibration of the molecular temperature with that of the environment. For all three anions, time constant ranging between 10 and 20 ps are found for this vibrational cooling. A slower 35 ps component is present with $\mathbf{D C A}^{\bullet-}$. This less efficient equilibration of $\mathbf{D C A}^{\bullet-}$ could be related to absence of $\mathrm{C} \equiv \mathrm{N}$ substituents in 2 and 6 positions, which leads to distinct vibrational modes for this anion compared to the other two. It should be noted that the solute and solvent dependence of intermolecular vibrational energy relaxation is still not fully understood.

These very short excited-state lifetimes are consistent with most of those reported previously with other open-shell ions in liquids. ${ }^{4,16-26,35-39}$ They are usually explained by the presence of conical intersection(s) between the $D_{1}$ and $D_{0}$ states that could be readily reached from the Franck-Condon $D_{1}$ state. ${ }^{25,27-30}$ TD-DFT calculations predict that, whereas the $\mathrm{D}_{2}-\mathrm{D}_{1}$ gap is less than $0.2 \mathrm{eV}$ at the $\mathrm{D}_{0}$ equilibrium geometry, it increases to about $0.7 \mathrm{eV}$ when going to the $\mathrm{D}_{1}$ geometry (Fig. S5, ESI $\dagger$ ). They also predict that, at the $\mathrm{D}_{2}$ equilibrium geometry, $\mathrm{D}_{2}$ becomes the lowest excited state. This points to the presence of $\mathrm{D}_{2} / \mathrm{D}_{1}$ conical intersection(s) along the relaxation pathway of $D_{2}$. According to these calculations, the energy of the $\mathrm{D}_{0}$ state at the $D_{2}$ and $D_{1}$ equilibrium geometries is only about 0.15 to $0.2 \mathrm{eV}$ above that at the $\mathrm{D}_{0}$ equilibrium geometry. However, these results do not preclude the existence of $D_{1} / D_{0}$ conical intersections, which remains the most probable origin of the very short excited-state lifetime of these anions. It should be noted that the presence of an electron in a anti-bonding molecular orbital makes these anions structurally more flexible than their neutral counterparts. According to the TD-DFT calculations, the $\mathrm{D}_{1} \leftarrow \mathrm{D}_{0}$ excitation involves electronic displacement from bonding to anti-bonding molecular orbitals (Section S4, ESI $\dagger$ ) and should, thus, lead to an enhanced flexibility of the excited anion, easing access to conical intersections. Proper detection of such intersections requires highlevel quantum-chemical calculations that would go beyond the scope of this investigation.

The excited-state lifetime of $\mathbf{D C A}^{\bullet-}$ is much shorter than the $4 \mathrm{~ns}$ value deduced from quenching measurements of $\mathbf{D C A}^{\boldsymbol{\bullet}^{-*}}$ produced by photoexcitation with a $5 \mathrm{~ns}$ pulse. ${ }^{33}$ This lifetime was estimated from the decrease of the $\mathbf{D C A}^{\bullet}{ }^{-}$bleach signal in the presence of an electron acceptor and by assuming a diffusioncontrolled quenching. However, at the high acceptor concentrations used in these experiment, up to $0.5 \mathrm{M}$, quenching is much more efficient than predicted assuming a pseudo-first order kinetics with the rate constant of diffusion. ${ }^{44,59,83}$ This is due to the contributions of the static and non-stationary stages of the quenching, which do not involve very significant diffusion of the reactants, and occur on a sub-ps to few tens ps timescales in nonviscous solvents. As these two non-equilibrium stages of quenching are dominant at such high concentrations, their neglect can lead to a strong overestimation of the lifetime of the excited reactant.

The very short excited-state lifetime of the radical anion of these cyanoanthracenes does not make them ideal systems for 
multiphoton photoredox catalysis. However, if the species to be reduced is at sufficiently high concentration, typically above $\sim 0.3 \mathrm{M}$, and if the driving force is sufficiently large, typically above $0.2 \mathrm{eV}$, the process could still occur relatively efficiently thanks to the static and non-stationary quenching regimes discussed above.

These lifetimes do not make the cyanoanthracenes very good candidates to detect the formation of excited ions upon highly exergonic photoinduced bimolecular ET, and thus to explain the discrepancy between the Weller experiment and Marcus theory. Among them, $\mathbf{D C A}^{\bullet-}$ is probably the best candidate as its $\mathrm{D}_{1}$ state exhibits a distinct absorption band around $460 \mathrm{~nm}$. Although DCA is the weakest electron acceptor, the ET quenching of its $S_{1}$ state by a strong electron donor like TMPD should be just exergonic enough to produce.

\section{Conclusions}

DCA $^{--}$has been suggested to be a well-suited radical anion for multi-photon redox catalysis based on a nanosecond excitedstate lifetime determined from indirect measurements. Our results reveal unambiguously that the excited-state lifetime of $\mathbf{D C A}^{\bullet-}$ and the radical anion of other cyanoanthracenes is shorter by three orders of magnitude. This is due to the presence of very efficient non-radiative pathway(s) to the $\mathrm{D}_{0}$ ground state. In this respect, these radical anions do not differ from most other organic open-shell ions, which are also characterised by very short excited-state lifetimes in room-temperature liquids. The ultrafast dynamics of these excited cyanoanthracene anions is most likely due to the presence of conical intersection(s) between the $\mathrm{D}_{1}$ and $\mathrm{D}_{0}$ states that are accessible from the Franck-Condon excited state, as found for many other radical ions. This property, which seems to be more a rule than an exception, most probably stems from the reduced rigidity of photoexcited radical ions compared to their neutral counterparts. This limits the choice of radical ions for multi-photon redox chemistry or at least imposes constraints on the experimental conditions. However, intermolecular photochemistry with cyanoanthracene radical anions should still be feasible if the quenching occurs in the static and non-stationary regimes. Further work is needed to confirm the viability of this approach.

\section{Data availability}

All data can be downloaded from https://doi.org/10.26037/ yareta:foqfztykprfwfalyhtksm56brq.

\section{Conflicts of interest}

There are no conflicts to declare.

\section{Acknowledgements}

The authors thank the Swiss National Science Foundation (grant 200020-184607) and the University of Geneva for financial support.

\section{Notes and references}

1 J.-C. Moutet and G. Reverdy, Nouv. J. Chem., 1983, 7, 105.

2 I. Ghosh, T. Ghosh, J. I. Bardagi and B. König, Science, 2014, 346, 725-728.

3 M. Fujitsuka and T. Majima, J. Photochem. Photobiol., C, 2018, 35, 25-37.

4 J. A. Christensen, B. T. Phelan, S. Chaudhuri, A. Acharya, V. S. Batista and M. R. Wasielewski, J. Am. Chem. Soc., 2018, 140, 5290-5299.

5 J. P. Barham and B. König, Angew. Chem., Int. Ed., 2020, 59, 11732-11747.

6 H. Kim, H. Kim, T. H. Lambert and S. Lin, J. Am. Chem. Soc., 2020, 142, 2087-2092.

7 F. Glaser, C. Kerzig and O. S. Wenger, Angew. Chem., Int. Ed., 2020, 59, 10266-10284.

8 D. K. Bohme, Chem. Rev., 1992, 92, 1487.

9 T. Snow, V. L. Page, Y. Keheyan and V. Bierbaum, Nature, 1998, 391, 259-260.

10 P. J. Sarre, J. Mol. Spectrosc., 2006, 238, 1-10.

11 G. Wenzel, C. Joblin, A. Giuliani, S. Rodriguez Castillo, G. Mulas, M. Ji, H. Sabbah, S. Quiroga, D. Peña and L. Nahon, Astron. Astrophys., 2020, 641, A98.

12 D. Rehm and A. Weller, Isr. J. Chem., 1970, 8, 259-271.

13 M. Koch, A. Rosspeintner, K. Adamczyk, B. Lang, J. Dreyer, E. T. J. Nibbering and E. Vauthey, J. Am. Chem. Soc., 2013, 135, 9843-9848.

14 A. I. Burshtein and A. I. Ivanov, Phys. Chem. Chem. Phys., 2007, 9, 396-400.

15 R. A. Holroyd and J. R. Miller, J. Phys. Chem. B, 2019, 123, 9206-9211.

16 Y. Huang and J. B. Hopkins, J. Phys. Chem., 1996, 100, 9585-9591.

17 J.-C. Gumy and E. Vauthey, J. Phys. Chem. A, 1997, 101, 8575-8580.

18 P. Brodard, A. Sarbach, J.-C. Gumy, T. Bally and E. Vauthey, J. Phys. Chem. A, 2001, 105, 6594-6601.

19 T. Häupl, R. Lomoth and L. Hammarström, J. Phys. Chem. A, 2003, 107, 435-438.

20 L. Zhao, R. Lian, I. Shkrob, R. Crowell, S. Pommeret, E. Chronister, A. Liu and A. Trifunac, J. Phys. Chem., 2004, 108, 25-31.

21 A. N. Okhrimenko, A. V. Gusev and M. A. J. Rodgers, J. Phys. Chem. A, 2005, 109, 7653-7656.

22 S. Pagès, B. Lang and E. Vauthey, J. Phys. Chem. A, 2006, 110, 7547-7553.

23 S. Samori, M. Fujitsuka and T. Majima, J. Phys. Chem. A, 2008, 112, 11312-11318.

24 S. Amarie, U. Förster, N. Gildenhoff, A. Dreuw and J. Wachtveitl, Chem. Phys., 2010, 373, 8-14.

25 J. Grilj, E. N. Laricheva, M. Olivucci and E. Vauthey, Angew. Chem., Int. Ed., 2011, 50, 4496-4498.

26 J. Grilj, P. Buchgraber and E. Vauthey, J. Phys. Chem. A, 2012, 116, 7516-7522.

27 K. F. Hall, M. Boggio-Pasqua, M. J. Bearpark and M. A. Robb, J. Phys. Chem. A, 2006, 110, 13591-13599. 
28 A. M. Tokmachev, M. Boggio-Pasqua, M. J. Bearpark and M. A. Robb, J. Phys. Chem. A, 2008, 112, 10881-10886.

29 A. M. Tokmachev, M. Boggio-Pasqua, D. Mendive-Tapia, M. J. Bearpark and M. A. Robb, J. Chem. Phys., 2010, 132, 044306.

30 K. S. Zinchenko, F. Ardana-Lamas, I. Seidu, S. P. Neville, J. van der Veen, V. U. Lanfaloni, M. S. Schuurman and H. J. Wörner, Science, 2021, 371, 489.

31 J. Eriksen and C. S. Foote, J. Phys. Chem., 1978, 82, 2659-2662.

32 J. Eriksen, K. A. Joergensen, J. Linderberg and H. Lund, J. Am. Chem. Soc., 1984, 106, 5083-5087.

33 M. Fujita, A. Ishida, T. Majima and S. Takamuku, J. Phys. Chem., 1996, 100, 5382-5387.

34 A. R. Cook, L. A. Curtiss and J. R. Miller, J. Am. Chem. Soc., 1997, 119, 5729-5734.

35 D. Gosztola, M. P. Niemczik, W. Svec, A. S. Lukas and M. R. Wasielewski, J. Phys. Chem. A, 2000, 104, 6545-6551.

36 M. J. Hope, M. P. Higlett, D. L. Andrews, S. R. Meech, I. D. Hands, J. L. Dunn and C. A. Bates, Chem. Phys. Lett., 2009, 474, 112-114.

37 M. Fujitsuka, T. Ohsaka and T. Majima, Phys. Chem. Chem. Phys., 2015, 17, 31030-31038.

38 C. Lu, M. Fujitsuka, A. Sugimoto and T. Majima, J. Phys. Chem. C, 2016, 120, 12734-12741.

39 C. Lu, M. Fujitsuka, A. Sugimoto and T. Majima, J. Phys. Chem. C, 2017, 121, 4558-4563.

40 C. Foote and J. Ericksen, J. Phys. Chem., 1978, 82, 2659-2882.

41 I. Gould, D. Ege, J. Moser and S. Farid, J. Am. Chem. Soc., 1990, 112, 4290.

42 E. Vauthey, J. Phys. Chem. A, 2001, 105, 340-348.

43 R. C. Vieira and D. E. Falvey, J. Phys. Chem. B, 2007, 111, 5023-5029.

44 M. Liang, A. Kaintz, G. A. Baker and M. Maroncelli, J. Phys. Chem. B, 2011, 116, 1370-1384.

45 A. Rosspeintner, G. Angulo and E. Vauthey, J. Am. Chem. Soc., 2014, 136, 2026-2032.

46 B. Wu, M. Liang, N. Zmich, J. Hatcher, S. I. Lall-Ramnarine, J. F. Wishart, M. Maroncelli and E. W. Castner, J. Phys. Chem. B, 2018, 122, 2379-2388.

47 A. J. Orr-Ewing, Struct. Dyn., 2019, 6, 010901.

48 R. A. Marcus and N. Sutin, Biochim. Biophys. Acta, Proteins Proteomics, 1985, 811, 265-322.

49 J. Eriksen, H. Lund and A. Nyvad, Acta Chem. Scand., 1983, B37, 459.

50 D. T. Breslin and M. A. Fox, J. Phys. Chem., 1994, 98, 408-411.

51 S. L. Mattes and S. Farid, J. Am. Chem. Soc., 1982, 104, 1454-1456.

52 M. A. Kellett, D. G. Whitten, I. R. Gould and W. R. Bergmark, J. Am. Chem. Soc., 1991, 113, 358-359.

53 A. Aster, G. Licari, F. Zinna, E. Brun, T. Kumpulainen, E. Tajkhorshid, J. Lacour and E. Vauthey, Chem. Sci., 2019, 10, 10629-10639.

54 J. S. Beckwith, B. Lang, J. Grilj and E. Vauthey, J. Phys. Chem. Lett., 2019, 10, 3688-3693.

55 T. Yanai, D. P. Tew and N. C. Handy, Chem. Phys. Lett., 2004, 393, 51-57.
56 C. Lee, W. Yang and R. G. Parr, Phys. Rev. B: Condens. Matter Mater. Phys., 1988, 37, 785-789.

57 M. J. Frisch, G. W. Trucks, H. B. Schlegel, G. E. Scuseria, M. A. Robb, J. R. Cheeseman, G. Scalmani, V. Barone, G. A. Petersson, H. Nakatsuji, X. Li, M. Caricato, A. V. Marenich, J. Bloino, B. G. Janesko, R. Gomperts, B. Mennucci, H. P. Hratchian, J. V. Ortiz, A. F. Izmaylov, J. L. Sonnenberg, D. Williams-Young, F. Ding, F. Lipparini, F. Egidi, J. Goings, B. Peng, A. Petrone, T. Henderson, D. Ranasinghe, V. G. Zakrzewski, J. Gao, N. Rega, G. Zheng, W. Liang, M. Hada, M. Ehara, K. Toyota, R. Fukuda, J. Hasegawa, M. Ishida, T. Nakajima, Y. Honda, O. Kitao, H. Nakai, T. Vreven, K. Throssell, J. A. Montgomery, Jr., J. E. Peralta, F. Ogliaro, M. J. Bearpark, J. J. Heyd, E. N. Brothers, K. N. Kudin, V. N. Staroverov, T. A. Keith, R. Kobayashi, J. Normand, K. Raghavachari, A. P. Rendell, J. C. Burant, S. S. Iyengar, J. Tomasi, M. Cossi, J. M. Millam, M. Klene, C. Adamo, R. Cammi, J. W. Ochterski, R. L. Martin, K. Morokuma, O. Farkas, J. B. Foresman and D. J. Fox, Gaussian-16 Revision C.01, Gaussian Inc., Wallingford CT, 2016.

58 S. U. Pedersen, T. B. Christensen, T. Thomasen and K. Daasbjerg, J. Electroanal. Chem., 1998, 454, 123-143.

59 A. Rosspeintner, M. Koch, G. Angulo and E. Vauthey, J. Am. Chem. Soc., 2012, 134, 11396-11399.

60 K. Kikuchi, M. Hoshi, T. Niwa, Y. Takahashi and T. Miyashi, J. Phys. Chem., 1991, 95, 38-42.

61 O. Laporte and W. F. Meggers, J. Opt. Soc. Am., 1925, 11, 459.

62 Z. Szakács and E. Vauthey, J. Phys. Chem. Lett., 2021, 12, 4067-4071.

63 I. H. M. van Stokkum, D. S. Larsen and R. van Grondelle, Biochim. Biophys. Acta, Proteins Proteomics, 2004, 1657, 82-104.

64 J. S. Beckwith, C. A. Rumble and E. Vauthey, Int. Rev. Phys. Chem., 2020, 39, 135-216.

65 A. Rosspeintner, B. Lang and E. Vauthey, Annu. Rev. Phys. Chem., 2013, 64, 247-271.

66 S. V. Feskov, V. A. Mikhailova and A. I. Ivanov, J. Photochem. Photobiol., C, 2016, 29, 48-72.

67 S. A. Kovalenko, R. Schanz, H. Hennig and N. Ernsting, J. Chem. Phys., 2001, 115, 3256-3274.

68 C. Weinert, B. Wezisla, J. Lindner and P. Vöhringer, Phys. Chem. Chem. Phys., 2015, 17, 13659-13671.

69 R. G. Fedunov, A. V. Plotnikova, A. I. Ivanov and E. Vauthey, J. Phys. Chem. A, 2017, 121, 471-481.

70 D. J. Nesbitt and R. W. Field, J. Phys. Chem., 1996, 100, 12735-12756.

71 J. Assmann, A. Charvat, D. Schwarzer, C. Kappel, K. Luther and B. Abel, J. Phys. Chem. A, 2002, 106, 5197.

72 C. G. Elles and F. F. Crim, Annu. Rev. Phys. Chem., 2006, 57, 273-302.

73 J.-M. L. Pecourt, J. Peon and B. Kohler, J. Am. Chem. Soc., 2001, 123, 10370-10378.

74 A. Pigliucci, G. Duvanel, L. M. L. Daku and E. Vauthey, J. Phys. Chem. A, 2007, 111, 6135-6145. 
75 C. T. Middleton, B. Cohen and B. Kohler, J. Phys. Chem. A, 2007, 111, 10460-10467.

76 O. Braem, T. J. Penfold, A. Cannizzo and M. Chergui, Phys. Chem. Chem. Phys., 2012, 14, 3513-3519.

77 A. Weller, Pure Appl. Chem., 1982, 54, 1885-1888.

78 M. Ue, K. Ida and S. Mori, J. Electrochem. Soc., 1994, 141, 2989-2996.

79 E. U. Condon, Phys. Rev., 1928, 32, 858-872.
80 H. L. Fragnito, J.-Y. Bigot, P. C. Becker and C. V. Shank, Chem. Phys. Lett., 1989, 160, 101.

81 D. M. Jonas, S. E. Bradforth, S. A. Passino and G. R. Fleming, J. Phys. Chem., 1995, 99, 2594-2608.

82 L. Monacelli, G. Batignani, G. Fumero, C. Ferrante, S. Mukamel and T. Scopigno, J. Phys. Chem. Lett., 2017, 8, 966-974.

83 M. Koch, A. Rosspeintner, G. Angulo and E. Vauthey, J. Am. Chem. Soc., 2012, 134, 3729-3736. 